\title{
A Theoretical Analysis of the Phase-Decompositions Based upon the Non-Linear Diffusion Equation
}

\author{
By Toru Miyazaki*, Takao Kozakai*, Shinya Mizuno** and Minoru Doi*
}

\begin{abstract}
A general form of the Fourier expression For the Cahn-Hilliard's flux equation is proposed, in which two kinds of the non-linear terms follow the linear term developed by Cahn, and computersimulations for the time developments of phase-decompositions in some supersaturated solid solutions are derived on the basis of the Fourier expression. The phase-decompositions proceed to form periodic zone-arrangements in the high solute alloys, while in the low solute alloys a few composition peaks sparsely distributed appear. The diffusion-behaviors simulated for the phase-decompositions seem to be essential for the basic understanding on the formations of microstructures.
\end{abstract}

(Received January 17, 1983)

Keyzords: phase-decomposition, diffusion equation, Fourier expression, computer simulation, spinodal decomposition, nucleation-growth, wave-synthesis

\section{Introduction}

Since the subject of phase-decomposition in supersaturated solid solutions has been recognized as one of the core topics of physical metallurgy, a great number of experimental works and theoretical proposals have been carried out. In the basic concept of phasedecomposition, solving the diffusion equation containing the non-linear term has been considered to be essential for analyzing the phasedecomposition process. Hillert ${ }^{(1)}$ derived a flux equation for one-dimensional diffusion and explained the periodicity of the microstructure produced by spinodal decomposition. $\mathrm{Cahn}^{(2)}$, using an interfacial energy and an elastic strain energy terms, developed a more flexible continuum model for an early stage of spinodal decomposition. Atempts to treat the later stages of spinodal decomposition have been also made by several authors such as Hillert ${ }^{(1)}, \mathrm{Cahn}^{(3)}$, Langer $^{(4)}$ and Tsakalakos ${ }^{(5)}$. However, because of difficulty of mathematical treatment for the diffusion equation, fairly serious assumptions were inevitably introduced in these analysis, that led the solutions valid only under limited conditions.

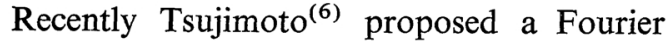

* Department of Metallurgical Engineering, Nagoya Institute of Technology, Nagoya 466, Japan.

** Graduate Student, Nagoya Institute of Technology. Present address: Toyota Motor Corporation, Toyota 471 , Japan. expression of the non-linear diffusion equation and succeeded in the kinetic pursuit of a zoneforming not only for the spinodal but also for the nucleation-growth decompositions. His works should be estimated to be highly valuable with respect to a lucid expression of the complex diffusion equation. However, the investigation was limited to an analysis for a single perturbation generated at the origin of distance-axis. Therefore, it is difficult to reflect his results on microstructures in actual alloys, because the microstructures must be formed as a result of zone-competitions with each other for the growth. Furthermore, Tsujimoto's analysis was derived on the basis of a polynomial expression of chemical free energy up to the 4th order of solute composition, which might be a fictitious form separating from the free energies of common alloys.

In the present work we first propose a general form of the Fourier expression of the non-linear diffusion equation so as to be applicable to the actual phase-decompositions, and then calculate time developments of phase-decompositions in several supersaturated solid solutions having various solute compositions.

\section{Theoretical Basis}

\section{The free energies of solid solutions and the interdiffusion coefficients}

We start by writing the free energy of the solid solution in the form of a polynomial of solute 
content $c$.

$$
f(c)=a_{n} c^{n}+a_{n-1} c^{n-1}+\cdots+a_{2} c^{2}+a_{1} c+a_{0} .
$$

This formalism approximates any curves of free energy for binary solid solutions. The free energy $f$ is related to the interdiffusion coefficient $\widetilde{D}$ as shown in eq. (2).

$$
\widetilde{D}=M\left(\partial^{2} f / \partial c^{2}\right),
$$

where $M$ is the mobility of solute atoms, assumed in this work to be constant. Substituting eq. (1) to eq. (2), we obtain definitely a relationship between $\tilde{D}$ and $c$. Here we practice, as an example, the analytical treatment on a polynomial free energy equation containing up to the 6th order term for simplicity of treatment. By defining composition variations $q$ to be $c-c_{\mathrm{A}}\left(c_{\mathrm{A}}\right.$ : average solute content), the interdiffusion coefficient $\tilde{D}$ is represented as a function of $q$;

$$
\begin{aligned}
\tilde{D}= & D_{0}+D_{1} q+D_{2} q^{2}+D_{3} q^{3}+D_{4} q^{4}, \\
D_{0}= & D_{4} / a_{6}\left[\left(a_{6} c_{\mathrm{A}}^{4}\right)+(2 / 3) a_{5} c_{\mathrm{A}}^{3}+(2 / 5) a_{4} c_{\mathrm{A}}^{2}\right. \\
& \left.+(1 / 5) a_{3} c_{\mathrm{A}}+(1 / 15) a_{2}\right], \\
D_{1}= & D_{4} / a_{6}\left[\left(4 a_{6} c_{\mathrm{A}}^{3}\right)+2 a_{5} c_{\mathrm{A}}^{2}+(4 / 5) a_{4} c_{\mathrm{A}}+(1 / 5) a_{3}\right], \\
D_{2}= & D_{4} a_{6}\left[\left(6 a_{6} c_{\mathrm{A}}^{2}+2 a_{5} c_{\mathrm{A}}+(2 / 5) a_{4}\right],\right. \\
D_{3}= & D_{4} / a_{6}\left[\left(4 a_{6} c_{\mathrm{A}}\right)+(2 / 3) a_{5}\right]
\end{aligned}
$$

and

$D_{4}=30 a_{6} M$.

The interdiffusion coefficient $\widetilde{D}$ is expressed by a polynomial equation of the compositionvariation $q$. The coefficients $D_{0}, D_{1}, D_{2}$ and $D_{3}$ are shown as functions of the composition $c$ and $D_{4}$. When the free energy is given by the general form shown in eq. (1), eq. (3) is written in a polynomial form up to $D_{n-2} q^{n-2}$ term.

Amit et al. ${ }^{(7)}$ proposed eq. (4) for the chemical free energy which represented a bysymmetric curve with composition.

$$
f(c)=-\zeta(2 c-1)^{2}+\xi(2 c-1)^{6},
$$

where $\zeta$ and $\xi$ are positive parameters deciding unevenness of the convex free energy curve. A relationship between the binodal composition $c_{\mathrm{e}}$ and the spinodal composition $c_{\mathrm{s}}$ in eq. (4) is given by eq. (5).

$$
5\left(2 c_{\mathrm{s}}-1\right)^{4}=\left(2 c_{\mathrm{e}}-1\right)^{4} .
$$

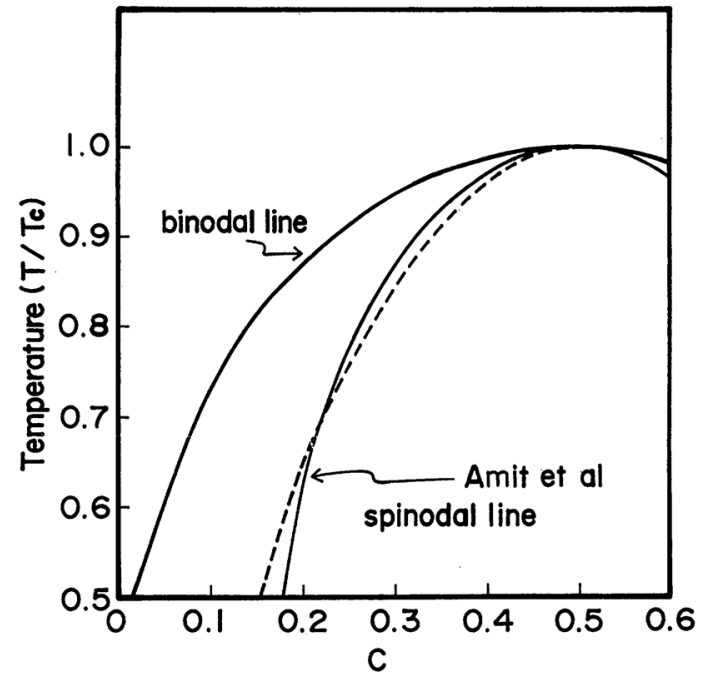

Fig. 1 A phase-diagram calculated on the basis of the free energies of eq. (4) proposed by Amit et al. and the regular solution-approximation. A dotted line and a thin solid line are spinodal lines for the regular solution-approximation and eq. (4) respectively. The binodal line is drawn common to the both free energies.

Figure 1 is a phase-diagram described based upon the free energies of eq. (4) and the regular solution-approximation, where the binodal line is drawn to common to the both free energies. The two spinodal lines are in considerably good coinc dence. When eq. (4) is adopted as the free energy equation, the interdiffusion coefficients $\widetilde{D}$ is concisely shown in eq. (6), instead of eq. (3).

$$
\begin{aligned}
& \widetilde{D}=D_{0}+D_{1} q+D_{2} q^{2}+D_{3} q^{3}+D_{4} q^{4}, \\
& D_{0}=-(1 / 16) D_{4}\left(C_{\mathrm{s}}^{4}-C^{4}\right) \\
& D_{1}=(1 / 2) D_{4} C^{3} \\
& D_{2}=(3 / 2) D_{4} C^{2} \\
& D_{3}=2 D_{4} C
\end{aligned}
$$

and

$$
D_{4}=1920 \xi M,
$$

where $C=2 c-1$. By assuming that $D_{4}=5.4 \times$ $10^{-20} \mathrm{~m}^{2} / \mathrm{s}^{\dagger}$ and the spinodal composition $c_{\mathrm{s}}$ is 0.17 , changes in the diffusion coefficients with composition $c$ are illustrated in Fig. 2. The constant term $D_{0}$ is negative in the spinodal

$\dagger$ Even if a separate value of $\mathrm{D}_{4}$ is used, phase-decompositions calculated are not affected in the behavior itself but in kinetics only. 


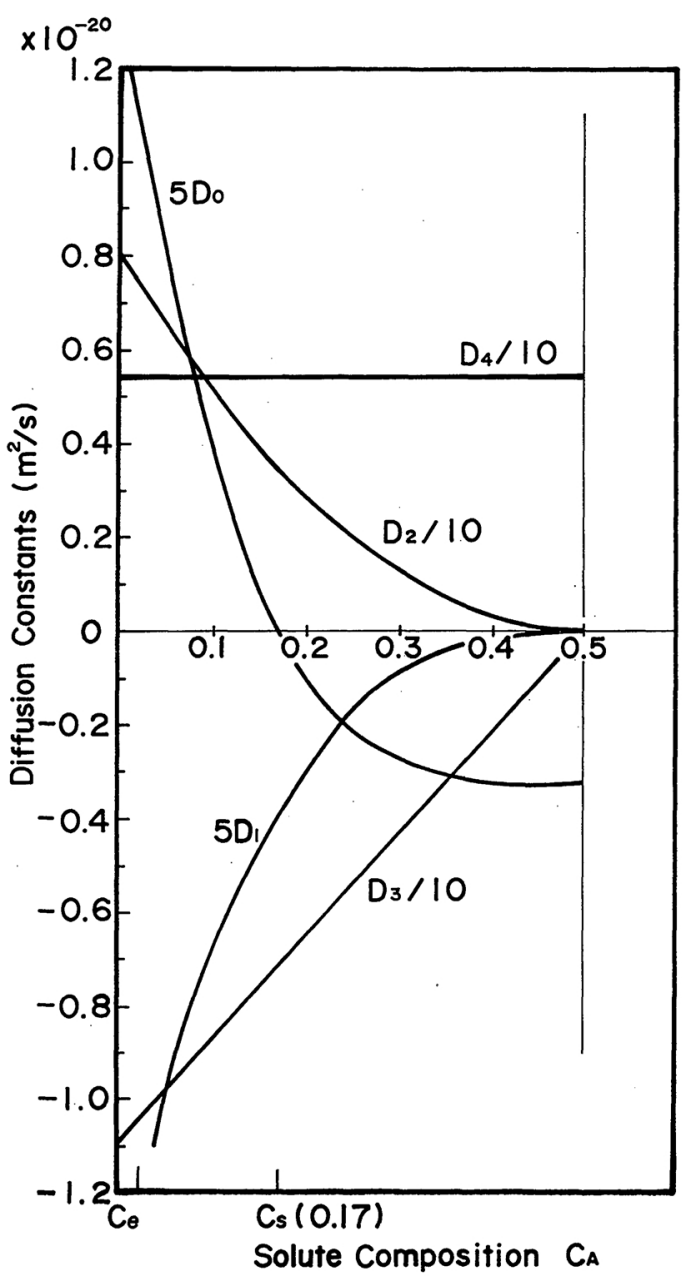

Fig. 2 Changes in diffusion coefficients $D_{0}, D_{1}, D_{2}$ and $D_{3}$ with the average solute composition $c_{\mathrm{A}}$ of a solid solution whose chemical free energy is given by eq. (4). The graph is by symmetric about the composition 0.5 . The spinodal composition $c_{\mathrm{s}}=0.17$ and 0.83 , and the equilibrium composition $c_{\mathrm{e}}=$ 0.02 and 0.98 .

region but positive in the binodal region. Absolute values of $D_{1}, D_{2}$ and $D_{3}$ become smaller -with increase of composition $c$ and zero at the centre of the miscibility gap.

\section{Diffusion equation}

We assume that diffusion behavior for phasedecomposition is given by eq. (7), which is usually called Cahn-Hilliard's flux equation ${ }^{(8)}$.

$$
\partial c / \partial t=\partial(\tilde{D} \partial c / \partial x) / \partial x-2 \tilde{K}\left(\partial^{4} c / \partial x^{4}\right),
$$

in which $\tilde{K}$ is the gradient energy coefficient.
Local deviation of composition from the average solute content at a location $x$ and at time $t$ is expressed as a sum of Fourier series, as is shown in eq. (8).

$$
q(x, t)=\Sigma Q_{h}(t) \exp (i h \beta x),
$$

where $Q_{h}(t)$ is an amplitude of a Fourier wave having the wave-number $h$ at time $t(h= \pm 1$, $\pm 2, \cdots, h \neq 0$ ) and $\beta$ is the reciprocal of length of the region considered $L$, defined by $\beta=2 \pi / L$. Substituting eq. (3) and eq. (8) into eq. (7) and arranging eq. (7), we derive a time evolution of the composition-variations as follows;

$$
\begin{aligned}
\partial q / \partial t= & -\beta^{2}\left\{\Sigma h^{2}\left(D_{0}+2 h^{2} \tilde{K} \beta^{2}\right) Q_{h} \exp [i h \beta x]\right. \\
& +D_{1} \Sigma \Sigma\left(h^{2}+h h^{\prime}\right) Q_{h} Q_{h^{\prime}} \exp \left[i\left(h+h^{\prime}\right) \beta x\right] \\
& +D_{2} \Sigma \Sigma \Sigma\left(h^{2}+2 h h^{\prime}\right) Q_{h} Q_{h^{\prime}} Q_{h^{\prime \prime}} \\
& \times \exp \left[i\left(h+h^{\prime}+h^{\prime \prime}\right) \beta x\right] \\
& +D_{3} \Sigma \Sigma \Sigma \Sigma\left(h^{2}+3 h h^{\prime}\right) Q_{h} Q_{h^{\prime}} Q_{h^{\prime \prime}} Q_{h^{\prime \prime \prime}} \\
& \times \exp \left[i\left(h+h^{\prime}+h^{\prime \prime}+h^{\prime \prime \prime}\right) \beta x\right] \\
& +D_{4} \Sigma \Sigma \Sigma \Sigma \Sigma\left(h^{2}+4 h h^{\prime}\right) Q_{h} Q_{h^{\prime}} Q_{h^{\prime \prime}} Q_{h^{\prime \prime \prime}} Q_{h^{\prime \prime \prime}} \\
& \left.\times \exp \left[i\left(h+h^{\prime}+h^{\prime \prime}+h^{\prime \prime \prime}+h^{\prime \prime \prime}\right) \beta x\right]+\cdots\right\} .
\end{aligned}
$$

In eq. (9) the first term on the right hand side, corresponding to the classical Cahn's spinodal theory ${ }^{(2)}$, shows a time evolution of the composition-variation $q(x, t)$, which arises from prosperity or decay of each Fourier component $Q_{h}$ with ageing time. The second and the Following terms show amplification-rates of fourier waves newly synthesized through interactions between the existent Fourier components: The second term concerns a synthesis of a wave having the wave-number $h+h^{\prime}$ from two waves of $h$ and $h^{\prime}$ respectively. Similarly the third term is on the rate of synthesizing a wave of $h+h^{\prime}+h^{\prime \prime}$ from three waves of $h, h^{\prime}$ and $h^{\prime \prime}$, respectively, and so on. Since the symbol $\Sigma$ in eq. (9) defines a summation for all wavenumber $h$, the 2 nd, 3rd, 4th and 5th terms of eq. (9) are mathematically equivalent to the one, two, three and four time convolutions of $Q_{h}$, respectively. Therefore, we rewrite the time dependence of $Q_{h}$ as follows;

$$
\begin{aligned}
& \partial Q(h) / \partial t \\
&=-(h \beta)^{2}\left[\left(D_{0}+2 h^{2} \widetilde{K} \beta^{2}\right) Q(h)+(1 / 2) D_{1} R(h)\right. \\
&+(1 / 3) D_{2} S(h)+(1 / 4) D_{3} T(h) \\
&\left.+(1 / 5) D_{4} U(h)+\cdots\right],
\end{aligned}
$$


where

$$
\begin{aligned}
& R(h)=\int_{-\infty}^{+\infty} Q(k) Q(h-k) d k \\
& S(h)=\int_{-\infty}^{+\infty} R(k) Q(h-k) d k \\
& T(h)=\int_{-\infty}^{+\infty} S(k) Q(h-k) d k
\end{aligned}
$$

and

$$
U(h)=\int_{-\infty}^{+\infty} T(k) Q(h-k) d k .
$$

If the free energy equation consists of the higher order terms over the 6 th, the sequent terms such as $D_{5}, D_{6}, \cdots$ follow after the $D_{4}$ term in eq. (10). This is a general form of the Fourier expression of the Cahn-Hilliard's diffusion equation. In eq. (10) the terms of odd number such as $D_{1}, D_{3}, D_{5}, \cdots$ give the rates of synthesizing new waves from $2,4,6, \cdots$ waves, respectively, and the signs of the generated components $Q_{h}$ are all the same. By contrast the terms of even number, that is, $D_{2}, D_{4}, D_{6}, \cdots$ give the rates of synthesizing from $3,5,7, \cdots$ waves, respectively, and the spectra generated have alternating signs. Roles of the two types of wave-synthesis on the time development of phase-decomposition have already been analyzed by Tsujimoto ${ }^{(6)}$, although his examination was restricted to the $D_{1}$ and $D_{2}$ synthesises.

On the basis of eq. (10) we are able to calculate time evolutions of the Fourier components $Q(h)$ by repeating eq. (11).

$$
Q_{t+\Delta t}(h)=\left(\partial Q_{t}(h) / \partial t\right) \Delta t+Q_{t}(h) .
$$

\section{Computer Simulations of Phase-Decompositions}

Here we represent time developments of phase-decomposition simulated for supersaturated solid solutions whose chemical free energies are given by eq. (4). Average compositions of the solutions are widely ranged from the centre of the miscibility gap $\left(c_{\mathrm{A}}=0.5\right)$ to the binodal region. A temperature 0.5 of the phasediagram shown in Fig. 1 was so chosen for the calculation that the coexisting compositions $c_{\mathrm{e}}$ came to 0.02 and 0.98 and the spinodal compositions $c_{\mathrm{s}}$ came to 0.17 and 0.83 . Composi- tion-variations before the computation were introduced by superimposing 100 sinusoidal composition-waves having various wave numbers and small amplitudes. When amplitudes of the initial waves $Q(h)$ are all positive, an extreamly high concentration is locally produced at a location, $x=0$, the origin of the distanceaxis, because the waves are all coincident in phase at the origin. Such a location may act as a particularly predominant site on the phasedecomposition. Therefore, the initial components $Q(h)$ were chosen not only to be positive but also to be negative so as to avoid such unrealistic perturbation. The same initial composition-variations are used throughout the simulations, which is shown in Fig. 3. The value of $\beta$ is chosen so small, $\beta=8.04 \times 10^{7} \mathrm{~m}^{-1}$, namely $78.1 \mathrm{~nm}$ in length, that many composition-peaks are realized in the region calculated. An extent of the wave-number $h$ during the calculation is restricted within 256 for convenience of computation. The wave-length of the wave $(h=256)$ is about $0.3 \mathrm{~nm}$, nearly equal to a unit-cell length of common alloys. The gradient energy coefficient $\tilde{K}$ is assumed to be $1.63 \times 10^{-41} \mathrm{~m}^{4} / \mathrm{s}$. The phase-decomposition calculated are now represented.

\section{Spinodal decomposition in the symmetric composition alloy $\left(c_{\mathrm{A}}=0.5\right)$}

Figure 3 shows developments of the composition-profiles with ageing for a case of $c_{\mathrm{A}}=0.5$. A thick full line indicated as $0 \mathrm{ks}$ in the figure shows the initial composition-variations. It is clearly seen from the figure that the heights of the composition-peaks increase with ageing time but stop exactly at the equilibrium composition $c_{\mathrm{e}}$ and then the peaks become rectangular. It should be noted that the peaks do not always appear at the location where solutes were locally concentrated in the initial composition variations. For instance, the peaks 2 nd, 5th and 7th from the right side arise at the locations whose initial compositions were less than the average solute content. Consequently, in the high composition alloys the phase-decomposition proceeds to form a periodic structure as a whole without respect to each location having initially high concentration. However, such independence becomes weaker with lowering 


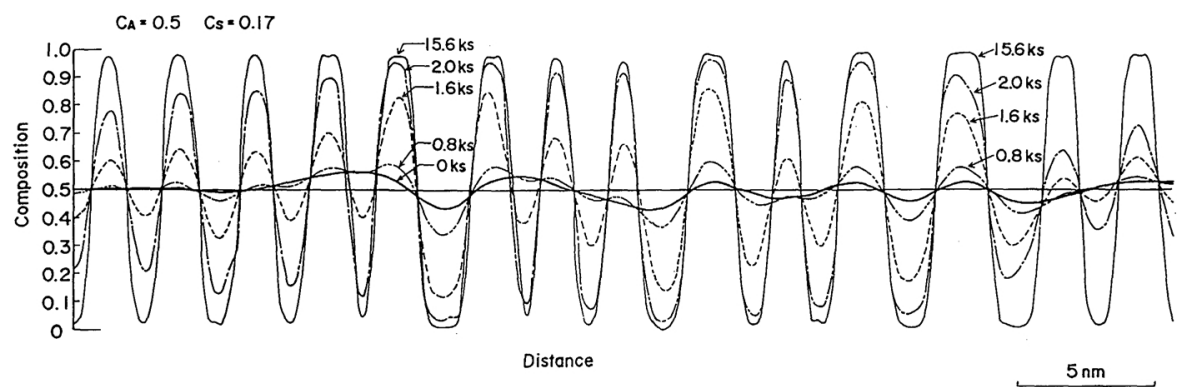

Fig. 3 Time developments of the composition-profiles with progress of ageing for the symmetric composition-alloy $\left(c_{\mathrm{A}}=0.5\right)$.

average composition of the alloys, particularly in the alloys near the spinodal line, which will be discussed later. The diffusion coefficients $D_{1}$, $D_{2}$ and $D_{3}$ are zero at the centre of the miscibility gap, as is shown in Fig. 2. Therefore, the phase-decomposition in Fig. 3 arises from the following two functions; amplifying the sinusoidal Fourier waves caused by the first term of eq. (10), that corresponds to the classical Cahn's spinodal theory, and making the peaks rectangular by the $D_{4}$ synthesis.

\section{Spinodal decomposition in the asymmetric composition alloy $\left(c_{\mathrm{A}}=0.3\right)$}

Figure 4 shows a decomposition process of a supersaturated solid solution in the alloy of $c_{\mathrm{A}}=0.3$. The number of the peaks in the figure is less than that of Fig. 3. The heights of some composition peaks increase temporarily but turn to decrease in the course of decomposition, as is seen in small peaks such as the 1st and 3rd from the left, that may result from a competitive growth between the peaks. The small peaks absorb solute atoms from the nearby matrix to grow up in the early stage of decomposition, while in the later stage the peaks must competitively absorb solute atoms from the other peaks since the solute atoms in the matrix have already been exhausted. Thus, the weak peaks are dissolved into the matrix and then absorbed into the nearby large peaks. This phenomenon is clearly viewed in the profile-changes of the 1st and 2nd peaks from the left of Fig. 4 where the servival peaks sidle up to the extinguished one. Such competitions for the growth, which may result in producing uniform distribution of zones, are often observed throughout the simulations, as shown later.

\section{Phase-decomposition at just inside spinodal line $\left(c_{\mathrm{A}}=0.2\right)$}

The case where the average composition of the alloy is inside but very close to the spinodal composition $c_{\mathrm{s}}$ is represented in Fig. 5. As is shown in the figure the composition-peaks further decrease in number but the periodicity still remains. In spite of a phase-decomposition in the spinodal region the large compositionpeaks accompany a so-called three-phasestructure which is said to be formed when the phase-decomposition proceeds in the nucleation-growth region. Such a phenomenon arises from the following situation; the first term of eq. (10) weakens while the $D_{1}, D_{2}$ and $D_{3}$

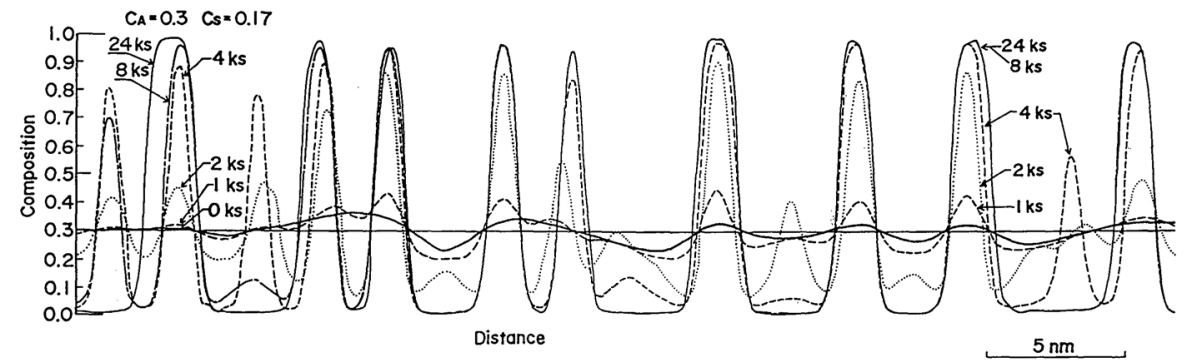

Fig. 4 Decomposition-process in a supersaturated solid solution of $c_{\mathrm{A}}=0.3$. 

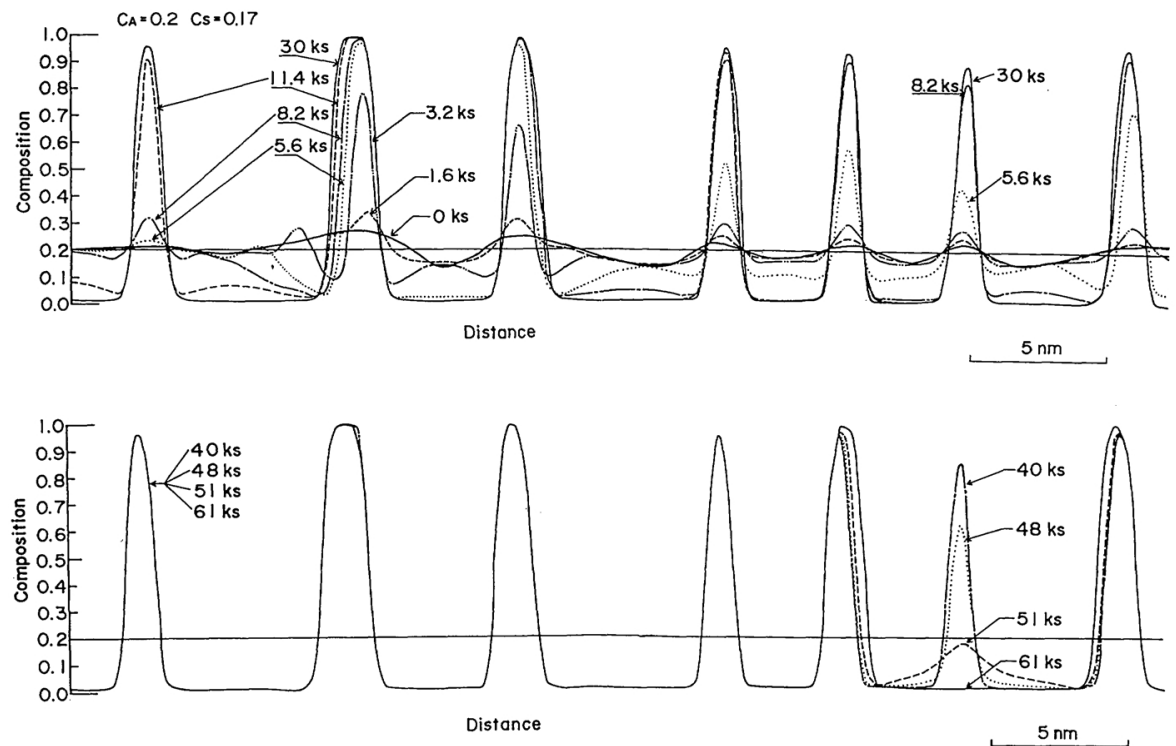

Fig. 5 Time developments of the composition-profiles with progress of ageing for the alloy just inside the spinodal line $\left(c_{\mathrm{A}}=0.2\right)$. The profiles are separately shown in upper and lower columns to avoid complicated overlapping of the many curves.

synthesises intensify their functions in the end of the spinodal region, that is, an inclination to the nucleation-growth type. A simulation for aggregation of zones is performed here, that is shown in the lower column of Fig. 5. The 2nd peak from the right is absorbed into the neighboring strong peaks, which is discussed later.

Since the curves of Fig. 5 are too complex to view details of the relative developments of the peaks, the composition-profiles are separately described for each ageing time in Fig. 6. It is obviously known from the rewritten profiles that the time developments of the peak-intensity greatly depend on the initial local solute concentrations in such an asymmetric composition alloy.

The process of the zone-aggregation is shown in the under part of Fig. 6. The peak-extinction seems to be determined not only by the peakintensity itself but also by a circumstance surrounding the peak, that is, by whether or not large peaks exist near the peak considered. This situation is comprehensible by comparing the time developments of the peak 2 nd from the right (called as peak 1) with the 1st from the left (peak 2) in Fig. 6. The local concentration of the peak 1 was initially so high that its intensity increased more rapidly during the early stage of ageing. However, as a stronger peak has existed near the peak 1 , the amplification-rate of that falls gradually off, and consequently the peak 1 is absorbed into the nearby stronger peaks through the competitive growth. On the other hand, the peak 2 having initially the lower concentration amplifies slowly at the early stage. But since the neighbor strong peak was fairly apart from the peak 2, it grows up to the equilibrium concentration $c_{\mathrm{e}}$ and still remains over a long duration for $61 \mathrm{ks}$. As above described, the zone-extinction proceeds in weak peaks existing near the strong peaks. According to such a consideration the next extinction may take place in the peak 4th from the left. Thus, the microstructure may aggregate keeping periodic zone-arrangements.

Figure 7 shows changes in amplitude of representative Fourier waves $Q_{h}$ with ageing time. In the early stage of ageing up to $10 \mathrm{ks}$ most of the components amplify to establish the periodic zone-arrangements of Fig. 6. After that, the components $Q_{h}$ are scarcely changed for a fairly long duration, which has been named a pseudo-equilibrium by Tsujimoto ${ }^{(6)}$, and then vary rapidly at about $50 \mathrm{ks}$. Such rapid changes in $Q_{h}$ result in the peak-extinction shown in Figs. 5 and 6. 


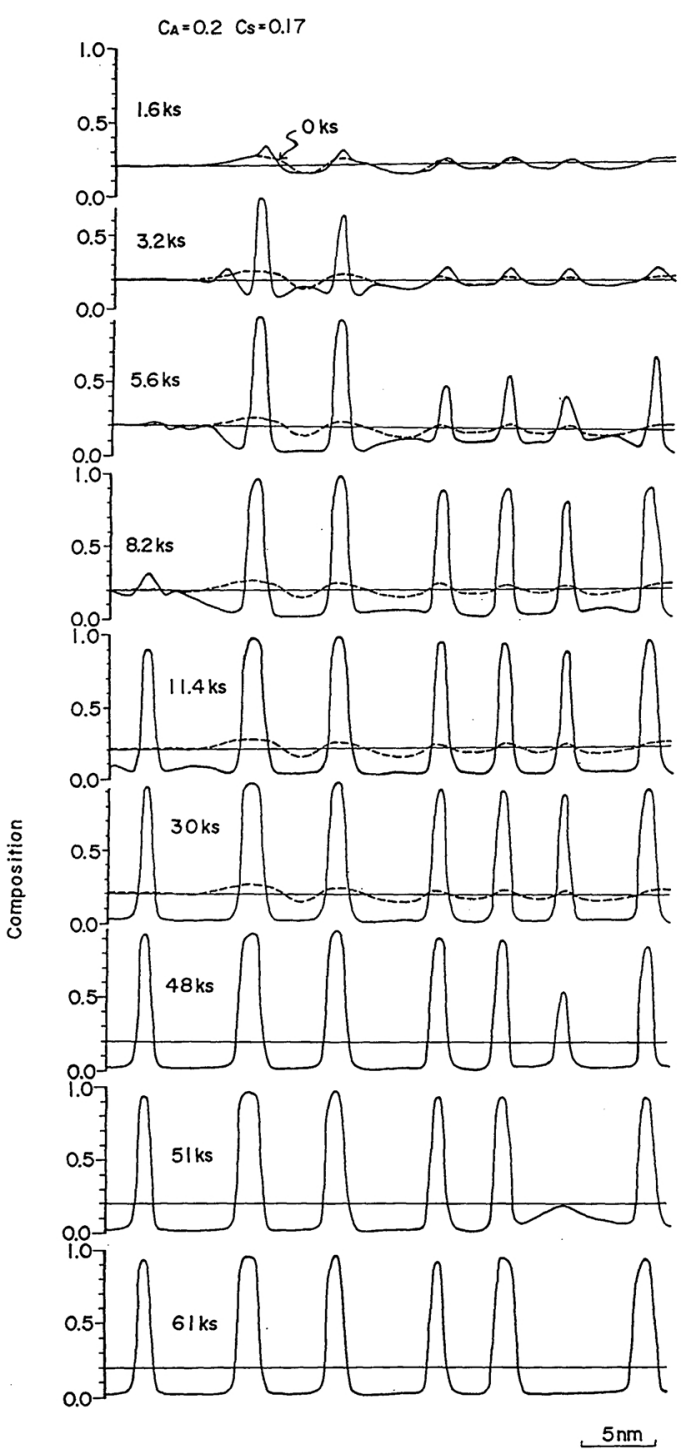

Fig. 6 The composition-profiles at each ageing time for the alloy $c_{\mathrm{A}}=0.2$, showing the time development of the phase-decomposition and the zone-extinction.

\section{Phase-decomposition at just on the spinodal line $\left(c_{A}=0.17\right)$}

Figure 8 represents a progress of phasedecomposition for the alloy $c_{\mathrm{A}}=0.17$. Three composition-peaks are only recognized on the locations where the local solute concentrations were initially high. Distributions of the peaks in Fig. 8 become no longer periodic. The phasedecomposition based upon the Cahn's classical spinodal theory never proceeds, because the diffusion coefficient $D_{0}$ is zero at the spinodal composition $c_{\mathrm{s}}$, as is shown in Fig. 2. However, the decomposition is virtually progressed, because the time evolution of amplitude arises in many Fourier waves throughout the $D_{1}, D_{2}, D_{3}$ and $D_{4}$ synthesisses.

\section{Phase-decomposition in the nucleation- growth region $\left(c_{\mathrm{A}}=\mathbf{0 . 1 5}\right)$}

Figure 9, a case where $c_{\mathrm{A}}=0.15$, shows that a peak is only formed at a location highly concentrated in the initial variations. It is clearly seen that the composition-peak, absorbing the solutes from the matrix by the down-hill diffusion, grows up to $c_{\mathrm{e}}$ and then increases in size with progress of ageing. This is a typical diffusion-behavior for the case of nucleation-growth types.

The competitive growth is also recognized in this case. The location just on the right hand side of the peak, whose initial composition was secondarily high, presents no compositionpeak, as is seen in Fig. 9. However, it should be noted that, because the solute concentration in that location exceeded locally the spinodal composition $c_{\mathrm{s}}$, a composition-peak must appear on that location if the location has singly existed in the initial composition variations without any other sites having higher solute concentrations ${ }^{\dagger}$. Therefore, it is concluded that such a disappearance of the peak which ought to have appeared is caused by a losing in competition for growth of the second site. Thus, even if the locations have a fairly high concentration initially, they can not always be sites for peakformation, but a few of them which overcome in the competition can generate the composition-peak. Such a competition for the peak-formation may imply the well-known experimental fact that G-P zones do not agglomerate but distribute uniformly in the matrix.

\section{Discussion}

On the basis of the simulation-results above described, the diffusion-behaviors in the phasedecompositions are summarized as follows;

$\dagger$ It was confirmed by the computer-simulation that a composition-peak appears for such a condition. 


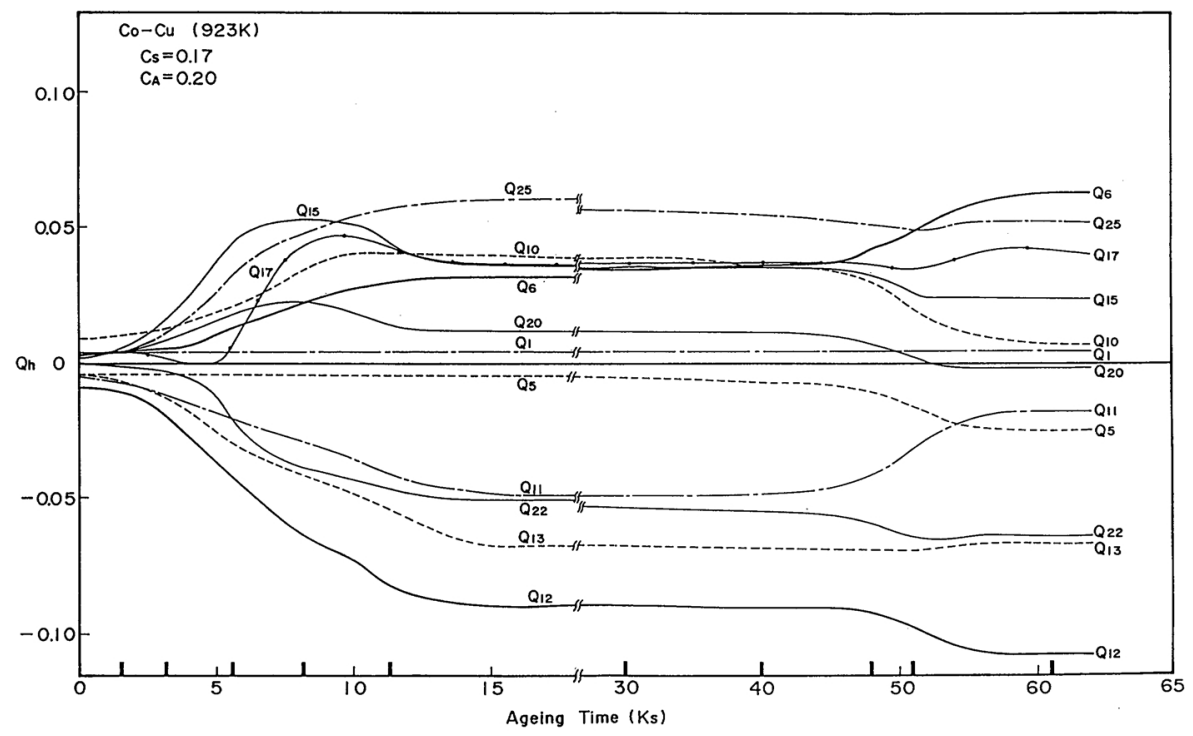

Fig. 7 Time dependences of amplitudes $Q_{h}$ of the representative Fourier waves. Thick full rods on the abscissa correspond to the ageing times of Fig. 6 respectively.

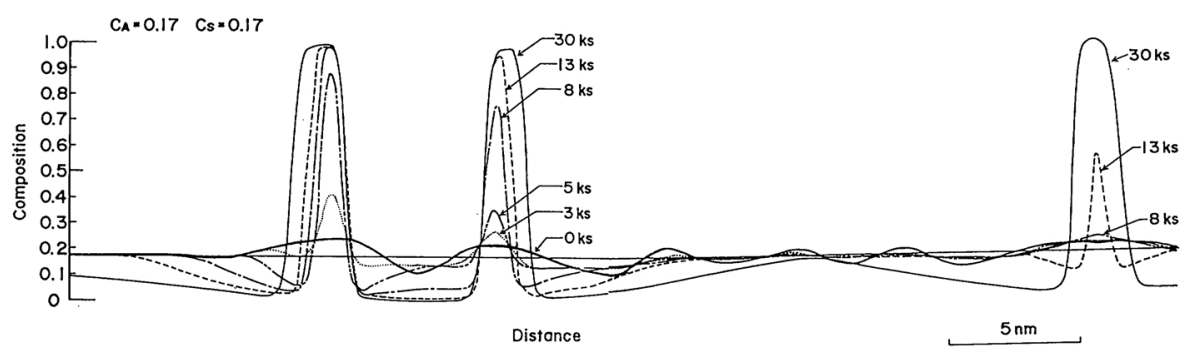

Fig. 8 A progress of phase-decomposition for the alloy $c_{\mathrm{A}}=0.17$, just equal to the spinodal composition $c_{\mathbf{s}}$.

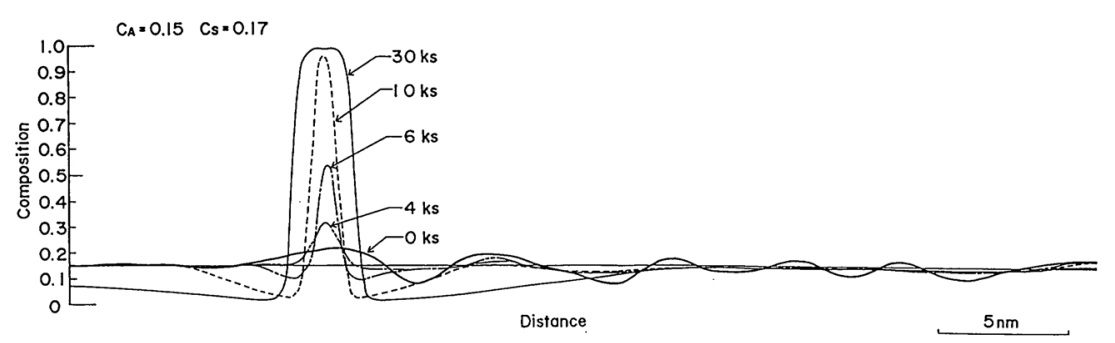

Fig. 9 Time developments of the composition-peak for the alloy whose average solute content $c_{\mathrm{A}}=\mathbf{0 . 1 5}$, showing a zone-formation in the nucleation-growth region of the miscibility gap.

in the high solute content alloys such as a symmetric alloy, the zones are nearly concurrently generated and grown up to form a periodic microstructure without strict corresponding to the local concentrations in the initial composition-variations. On the contrary, in the low concentration alloys the rates of amplification depend greatly on the degrees of the local concentrations in the initial composition variations. Therefore, the zones can be viewed so as to increase gradually in number with progress of ageing, that is a characteristic of the nucleationgrowth in phase-decomposition.

According to the present theory, it is con- 
sidered that the coarsening-rate in the periodic structures depends on the average composition of alloys; the higher the alloy composition is, the lower the rate becomes. The extinction of zones resulting in the coarsening of the structure arises from the $D_{1}, D_{2}, D_{3}$ and $D_{4}$ synthesises. The diffusion constants $D_{1}, D_{2}$ and $D_{3}$ become extreamly small in the central part of miscibility gap, as is shown in Fig. 2. Therefore, the coarsening-rates of the periodic structure must become low with increase in composition of the alloys. Such an inference seems to be verified by the experimental fact that the coarsening-rates of the modulated structure in many alloys are lower in the higher composition-alloys ${ }^{(9)}$.

Finally, we discuss on the diffusionbehaviors, when the initial composition-variations is completely zero. According to the present theory, the phase-decompositions do not progress at all for such a condition, even if in the symmetric composition alloy, because the phase-decomposition theories based upon the diffusion equation are applicable only when the composition variations exist initially. However, it is expected that the composition-variations are always introduced into the supersaturated solid solutions when the alloys are quenched, probably originated from the thermal fluctuations at the homogenizing temperature ${ }^{(10)}$. Furthermore, even if no composition-variations are quenched-in, the composition fluctuations may arise from the Brownian motions of atoms at the ageing temperature. Consequently, the theoretical treatment in the present work is certainly realistic for the phase-decomposition.
The theoretical analysis presented here is restricted to the one-dimensional phase-decomposition in the alloy having a symmetric free energy curve with composition. Nevertheless, the simulation-results are quite suggestive for the basic understanding of the formation of microstructure. Further understanding of the phase-decomposition may be introduced by the theoretical examinations based upon the two and three-dimensional diffusion equations, although laborious calculations are required.

\section{Acknowledgements}

The authors would like to thank Dr. A. Iwata, Department of Information Engineering of Nagoya Institute of Technology, for his discussion on the computer programming. This work was supported in part by a Grant-in-Aid for Scientific Research from the Ministry of Education, Science and Culture, Japan.

\section{REFERENCES}

(1) M. Hillert: Acta Met., 9 (1961), 525.

(2) J. W. Cahn: Acta Met., 9 (1961), 795; 10 (1962), 179.

(3) J. W. Cahn: Acta Met., 14 (1966), 1685.

(4) J. S. Langer: Acta Met., 21 (1973), 1649.

(5) T. Tsakalakos: Scripta Met., 15 (1981), 255.

(6) T. Tsujimoto: Trans. JIM, 21 (1980), 458; 22 (1981), 127; 22 (1981), 614.

(7) D. Amit: Phys. Lett., A26 (1968), 466; S. Fisk and B. Widom: J. Chem. Phys., 50 (1969), 3219.

(8) J. E. Hilliard: Phase Transformations (Chapter 12), Metals Park, Ohio, ASM, (1970).

(9) For instance, R. W. Carpenter: Acta Met., 15 (1967), 1567.

(10) For instance, E. L. Huston, J. W. Cahn and J. E. Hilliard: Acta Met., 14 (1966), 1053. 